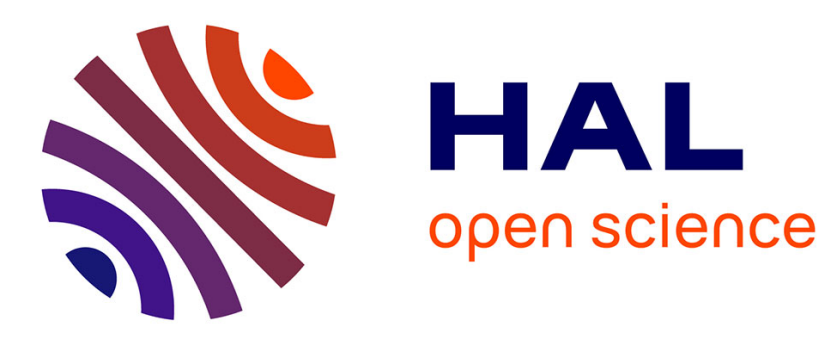

\title{
Title: Consequences of population structure for sex allocation and sexual conflict
}

Leonor R Rodrigues, Mario Torralba Sáez, João Alpedrinha, Sophie Lefèvre, Muriel Brengues, Sara Magalhães, Alison Duncan

\section{To cite this version:}

Leonor R Rodrigues, Mario Torralba Sáez, João Alpedrinha, Sophie Lefèvre, Muriel Brengues, et al. Title: Consequences of population structure for sex allocation and sexual conflict. Journal of Evolutionary Biology, 2021, 34, 3, pp.525-536. 10.1111/jeb.13755 . hal-03049532

\section{HAL Id: hal-03049532 \\ https://hal.science/hal-03049532}

Submitted on 9 Dec 2020

HAL is a multi-disciplinary open access archive for the deposit and dissemination of scientific research documents, whether they are published or not. The documents may come from teaching and research institutions in France or abroad, or from public or private research centers.
L'archive ouverte pluridisciplinaire HAL, est destinée au dépôt et à la diffusion de documents scientifiques de niveau recherche, publiés ou non, émanant des établissements d'enseignement et de recherche français ou étrangers, des laboratoires publics ou privés. 


\section{Title: Consequences of population structure for sex allocation and sexual conflict}

2 Authors: Leonor R. Rodrigues ${ }^{1} \dagger$, Mario Torralba Sáez ${ }^{2 \dagger}$, João Alpedrinha ${ }^{1}$, Sophie Lefèvre ${ }^{2}$,

3 Muriel Brengues $^{3}$, Sara Magalhães ${ }^{1}$ and Alison B. Duncan ${ }^{2 *}$.

4 †These authors contributed equally to the manuscript.

5 Author institutional affiliations:

6 1. cE3c: Centre for Ecology, Evolution, and Environmental Changes, Faculty of Sciences,

7 University of Lisbon, Edifício C2, 38 piso, 1749-016 Lisboa, Portugal

8 2. ISEM : Institut des Sciences de l'Évolution, Université de Montpellier, CNRS, IRD, EPHE,

9 CC065, Place Eugène Bataillon, 34095 Montpellier Cedex 05, France

10 3. IRCM : Institut de Recherche en Cancérologie de Montpellier, INSERM, Univ. Montpellier,

11 ICM, Montpellier, France 


\section{Abstract}

Both sex allocation and sexual conflict can be modulated by spatial structure. However, how the interplay between the type of dispersal and the scale of competition simultaneously affects these traits in sub-divided populations is rarely considered.

19 We investigated sex allocation and sexual conflict evolution in meta-populations of the spider mite Tetranychus urticae evolving under budding (pairing females from the same patch) or random

21 (pairing females from different patches) dispersal and either local (fixed sampling from each 22 subpopulation) or global (sampling as a function of subpopulation productivity) competition.

24 Females evolving under budding dispersal produced less female-biased offspring sex ratios than those from the random dispersal selection regimes, contradicting theoretical predictions. In contrast, the scale of competition did not strongly affect sex allocation. Offspring sex ratio and female fecundity were unaffected by the number of mates, but female fecundity was highest when their mates evolved under budding dispersal, suggesting these males inflict less harm than those evolving under random dispersal.

31 This work highlights that population structure can impact the evolution of sex allocation and sexual conflict. Moreover, selection on either trait may reciprocally affect the evolution of the other, for example via effects on fecundity.

Keywords: local mate competition, hard and soft selection, experimental evolution, budding dispersal, scale of competition, Tetranychus urticae. 
Introduction

Many organisms exist in structured populations, sub-divided into patches, that are linked and shaped by demographic factors such as dispersal. The frequency and type of dispersal can determine whether interactions are more likely to occur among related or unrelated individuals (Hamilton, 1964, Bulmer, 1986, Queller, 1992, Rousset, 2004, Courteau \& Lessard, 2000, West, 2009). For instance, if dispersal is limited, such that only some individuals disperse, the probability of interactions among genetically related individuals in a patch increases compared to populations in which all individuals disperse (Taylor, 1992, Wilson et al., 1992, Taylor \& Crespi, 1994, Hamilton, 1964). However, if individuals disperse in groups from the same patch (i.e., if there is budding dispersal), interactions among genetically related individuals can be maintained, even if dispersal rates are high (Gardner et al., 2009, Gardner \& West, 2006, Lehmann et al., 2006, Lehmann \& Rousset, 2010, Aviles, 1993).

Dispersal frequency and timing also influence the scale of competition. For example, high dispersal, and dispersal occurring prior to the competitive interaction, leads to global competition, in which individuals compete with equal probability with others in the population (West et al., 2002a, Griffin et al., 2004, Taylor, 1992). In contrast, limited dispersal, and/or dispersal occurring after the competitive interaction, is associated with local competition (i.e., competition within the natal patch) (West et al., 2002a, Griffin et al., 2004, Taylor, 1992, Frank, 1998, Wilson et al., 1992). Therefore, the type, frequency and timing of dispersal can have a significant impact on the scale at which competitive interactions occur.

In turn, both relatedness and the scale of competition can affect sex allocation - the differential investment into male and female offspring. Indeed, in subdivided populations, sex allocation theory predicts an offspring sex-bias towards the sex for which local competition 
between kin is less intense (Wilson et al., 1992, Hamilton, 1967, Charnov, 1982). For example, more female-biased offspring sex ratios are predicted when males compete locally on their natal patch for mates, and mated females disperse and compete globally for new patches (Hamilton, 1967, Taylor, 1981, Herre, 1985). If there is budding dispersal, relatedness among the offspring of foundresses increases, exacerbating local competition between related males for mates, thus selecting for even more female-biased sex ratios (Gardner et al., 2009, Aviles, 1993). However, if the proportion of individuals dispersing is limited, and females compete locally for resources, competition becomes intense for both sexes and selection favours a more balanced offspring sex ratio (Table S1; Courteau \& Lessard, 2000, Taylor \& Crespi, 1994, Frank, 1985, Herre, 1985, Bulmer, 1986, Frank, 1986). A few empirical studies to date have investigated the consequences of budding dispersal (Kummerli et al., 2009), or disentangled the relative effects of the scale of competition and relatedness (Griffin et al., 2004) on the evolution of kin-selected behaviours, but none have disentangled the effect of these two factors on sex allocation.

Population structure is also predicted to impact the evolution of sexual conflict, i.e., asymmetric reproductive interests between mating partners (Pizzari et al., 2015, Bourke, 2009, Faria et al., 2020). Competition for mates between males often involves strategies that are harmful to females reducing their survival and reproductive output (Arnqvist \& Rowe, 2005, Parker, 2006, Wigby \& Chapman, 2004, Crudgington et al., 2005). However, under global competition, when interactions occur among kin, reduced harming behaviour in males is expected to be selected for, as harm reduces patch productivity (Pizzari et al., 2015, Pizzari \& Gardner, 2012, Rankin, 2011). A number of empirical studies are compatible with this prediction (Lukasiewicz et al., 2017, Carazo et al., 2014, Hollis et al., 2015, Le Page et al., 2017), but see (Chippindale et al., 2015). For instance, in the fruit fly Drosophila melanogaster, females repeatedly exposed to related, as 
opposed to unrelated, males presented a higher lifetime reproductive success (Carazo et al., 2014).

84 The evolution of sexual conflict may also depend on the frequency (Eldakar \& Wilson, 2011) and

85

86

87 type of dispersal (Faria et al., 2020). Because random dispersal reduces relatedness among competitors, it is predicted to increase the intensity of sexual conflict (Faria et al., 2015, Rankin, 2011). This may be alleviated by budding dispersal, which maintains interactions among kin, reducing sexual conflict and harm inflicted to females, hence increasing their fecundity (Faria et al., 2020).

Although population structure is predicted to affect sex allocation and sexual conflict (Bourke, 2009), no study to date has disentangled how the type of dispersal and the scale of competition impacts the evolution of both within the same set-up. This is at odds with the fact that evolution under different population structures may simultaneously impact sex allocation and sexual conflict in a non-independent manner, highlighting the need to integrate studies on these traits (Chapman, 2009, Schärer \& Janicke, 2009). For instance, changes in sex allocation may result in the production of more or fewer individuals of each sex, which impacts sexual conflict. At the same time, sexual conflict may impact the number of offspring produced (Carazo et al., 2014, Lukasiewicz et al., 2017), which may in turn influence sex allocation (Stubblefield \& Seger, 1990). This is supported by studies showing that multiple mating can impede optimal sex allocation in the parasitoid wasp Nasonia vitripennis (Boulton et al., 2019, Boulton \& Shuker, 2015).

Here, we uncover the effects of the type of dispersal and the scale of competition on the evolution of sex allocation and sexual conflict in the spider mite Tetranychus urticae. Previous work in this species has shown the evolution of sex allocation, and conflict between the sexes over the optimal offspring sex-ratio, in populations evolving under panmixy or local mate competition 
106 (Macke et al., 2011, Macke et al., 2014). In addition, it is known that in this species only the sperm

107 from the first copulation fertilises a female's eggs (Rodrigues et al., 2020). Still, multiple mating 108 is common in this species, imposing costs in females by reducing the proportion of daughters as 109 well as female fecundity (Rodrigues et al., 2020, Macke et al., 2012). This reduction in female 110 reproductive output is thought to be a consequence of strong competition among males and, thus, 111 evidence of male harm due to sexual conflict (Rodrigues et al., 2020).

112 In a fully crossed design, using experimental evolution, we placed replicate populations of 113 T. urticae in 4 selection regimes with either local or global competition, and random versus 114 budding dispersal. This design enabled us to follow evolution of both sex ratio and sexual conflict 115 under different population structures. We expect sex allocation evolution under global competition 116 to be towards more female biased offspring sex ratios than under local competition, due to reduced 117 competition between related females locally in the patch. Furthermore, global competition, when 118 coupled with budding dispersal, should produce the most female biased offspring sex ratios (see 119 Table S1 for precise predictions for sex allocation under our different selection regimes). In contrast, as there is no mixing under local competition with budding dispersal, we do not expect 121 selection to occur in this treatment. Sexual conflict should also evolve differently in the different selection regimes. We predict that female harm inflicted by males, expressed as reduced fecundity, will be diminished when males evolve under budding dispersal, where interactions occur among kin. 


\section{Material and Methods}

128

129

130

131

132

133

134

135

136

137

138

139

140

141

142

143

144

145

146

147

148

\section{Biological model}

The two-spotted spider mite, T. urticae Koch (Acari: Tetranychidae), is a generalist herbivore with a host range of over 1100 plant species (Migeon \& Dorkeld, 2019, Helle \& Sabelis, 1985). T. urticae has an arrhenotokous haplodiploid life cycle ( $\sim 14$ days egg - adult at $\left.20-25^{\circ} \mathrm{C}\right)$ : sons develop from unfertilised, haploid eggs and daughters from fertilised, diploid eggs. In this species, females are the dispersing sex, whereas males generally remain on their natal patch with mating occurring before female dispersal (Kennedy \& Smitley, 1985, Mitchell, 1973). We report tertiary sex ratios (adult males divided by the total number of adult offspring) as males and females can only be distinguished as adults using microscopy: males are smaller than females and possess a pointed abdomen.

\section{Population origins}

In 2013, 10 different $T$. urticae populations were collected and separately maintained on bean plants at $25 \pm 2{ }^{\circ} \mathrm{C}$, with a $16 \mathrm{~h}$ light: $8 \mathrm{~h}$ dark cycle at the University of Lisbon. These populations comprised seven populations from Portugal (Lou, DC, AMP, DF, CH, COL and RF), two from Spain (Albe and Alro) and one from France (FR) (Zélé et al., 2018). All populations were treated with antibiotics to ensure that they were free of bacterial endosymbionts, known to be sex ratio distorters (Breeuwer, 1997). The sex ratio of each individual population ranges from 0.22 to 0.40 (Zélé et al., 2020). In November 2015, more than 50 females from each of the 10 populations were transferred to the University of Montpellier and mixed to form a genetically diverse population to seed the experiment (hereafter called the 'ancestral population'). This newly mixed population was maintained on 12 whole bean plants (variety: Pongo) in a plastic box (395 mm length x $335 \mathrm{~mm}$ 
width $\times 323 \mathrm{~mm}$ height) at $25^{\circ} \mathrm{C}$ with a $16 \mathrm{~h}$ light: $8 \mathrm{~h}$ dark cycle. Each week, old plants were removed and replaced with young, un-infested plants. All bean plants used to maintain mite populations and for all experiments described below were grown from seeds in an isolated, herbivore-free room at $23 \pm 1{ }^{\circ} \mathrm{C}$ with a photoperiod of $12 \mathrm{~h}$ light: $12 \mathrm{~h}$ dark at the University of Montpellier.

Fourteen days before starting the experiment, 10 independent groups of 40 females were haphazardly sampled from the ancestral population and put on a patch (10-15 bean leaves placed together) on water-saturated cotton wool to lay eggs. This allowed maternal effects to be equalised and ensured that females seeding the experiment were of the same age. Two weeks later, when mites of the following generation had reached adulthood, all 10 groups were mixed, and the newly emerged mated adult females were haphazardly assigned to the different selection regimes.

\section{Establishment and maintenance of selection regimes}

The impact of different types of dispersal (budding versus random) and scales of competition (local versus global) on the evolution of sex allocation and sexual conflict in T. urticae was investigated using a fully crossed experimental design (Figure 1): 1) global competition, budding dispersal ('Global Budding', GB), 2) global competition, random dispersal ('Global Random', GR), 3) local competition, budding dispersal ('Local Budding', LB) and 4) local competition, random dispersal ('Local Random', LR). Each regime was replicated three times (GB-1, GB-2, GB-3, GR-1, GR-2, GR-3, LB-1, LB-2, LB-3, LR-1, LR-2 and LR-3).

For each replicate population, each generation comprised a total of 96 mated adult females, being assigned in pairs to 48 bean leaf patches $\left(4 \mathrm{~cm}^{2}\right.$ each, corresponding to a very mild infestation level, thus low competition) placed on water-saturated cotton wool in a plastic box ( $255 \mathrm{~mm}$ length 


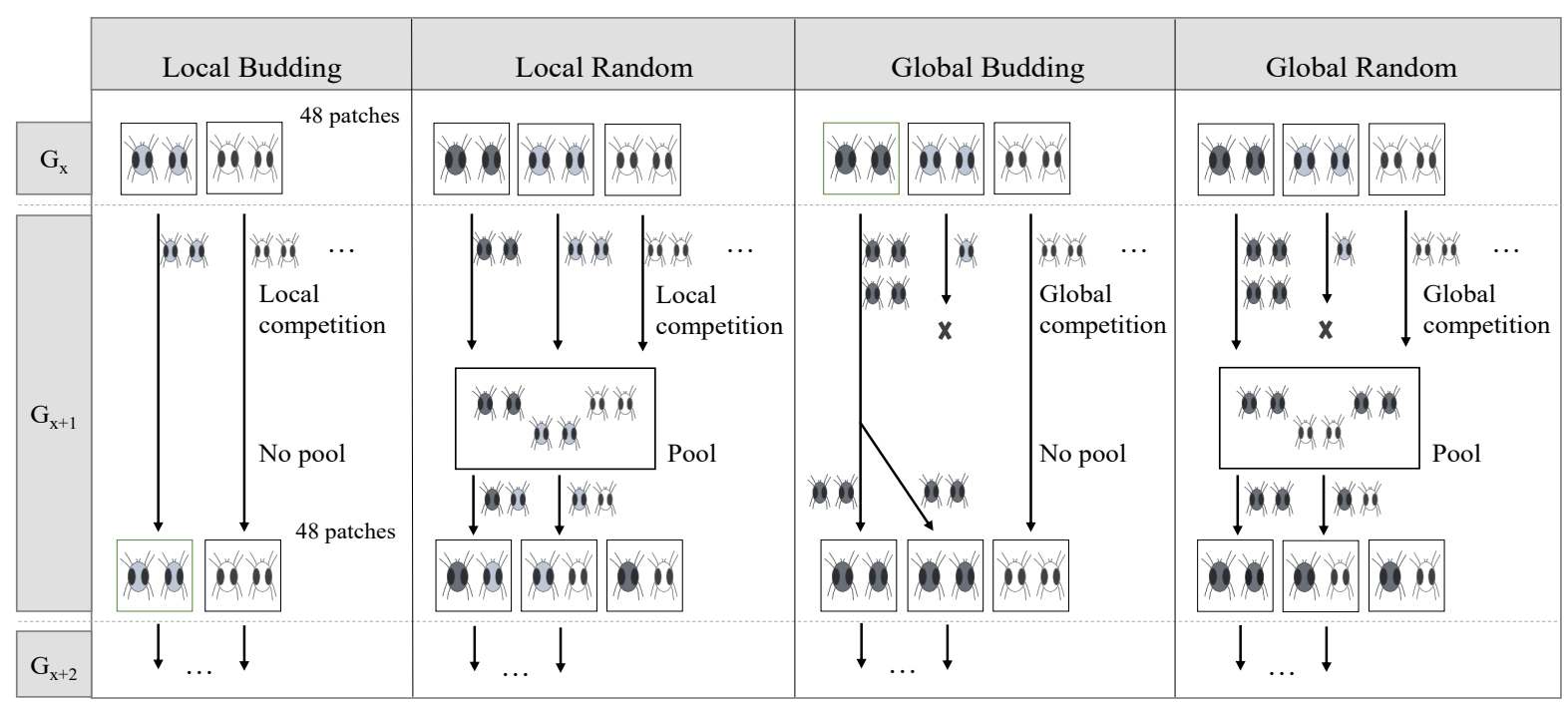

171

172 Figure 1. Description of the selection regimes. Four selection regimes were established and maintained for 33 generations, each with three experimental replicates. In 'Local Budding', 2

174 females from each of the 48 patches (squares) were transferred in pairs to a new patch for the next 175 generation $\left(\mathrm{G}_{\mathrm{x}+1}\right)$. In 'Local Random', an equal number of females $(2-4$; the number was adjusted 176 each generation to account for mortality) from each patch were pooled together on a large common 177 leaf patch ('mixing patch', rectangle), from which females were subsequently haphazardly 178 transferred in pairs to 48 new patches. In 'Global Budding', the number of adult females per patch 179 was counted before each transfer to calculate fecundity relative to that of the other 47 patches in 180 the replicate. Each patch contributed with a number of female pairs, to the following generation, proportional to its relative fecundity. In 'Global Random' all 48 patches were placed on a 'mixing patch' onto which females could disperse for $\sim 4$ hours, after which adult females were haphazardly transferred in pairs to 48 new patches for the next generation. Related females within a treatment are denoted as the same shade of grey.

$186 \times 183 \mathrm{~mm}$ width $\times 77 \mathrm{~mm}$ height). Mating occurred on the natal patch among the offspring of the 1872 females, before mated daughters dispersed. A single male is capable of fertilising up to 15 188 females in a single day (Krainacker \& Carey, 1989), hence the number of males available to 
189 fertilise all females on a patch was not limiting. All replicates from all regimes were maintained 190 in the same conditions, the only difference being how populations were mixed and transferred to 191 new patches at each generation (Figure 1). Note that such variation in population structure 192 inherently affects the effective population size and thus the degree of inbreeding in populations. In the budding dispersal regimes, females were always transferred with another female from the same patch to form the next generation. In contrast, in the random dispersal regimes, females from different patches were placed together on a 'mixing patch' (10 bean leaves placed together) before being transferred, in haphazardly chosen pairs, to a new patch. Local competition was imposed by letting an equal number of adult females per patch seed the next generation $(2-$ 4 females per patch in 'Local Random'; adjusted each generation to accommodate mortality). Under global competition, relative patch productivity (the total number of daughters produced compared to that of the other patches within the replicate) determined the number of female adult offspring transferred to the next generation: in the 'Global Random' regime, all 48 patches were placed on a 'mixing patch' onto which adult females dispersed (patches with more female offspring having a higher representation on the 'mixing patch') before being transferred in pairs; in the 'Global Budding' regime, the number of adult females on each patch was counted to calculate relative fecundity (i.e. dividing the number of females per patch by the total number of females across the 48 patches), so that patches with the most offspring contributed more pairs of 207 females to the next generation. Due to the time taken for each transfer, transfers from one generation to the next were done over 1, 2 or 3 days. When done over more than one day, at least one replicate population from each regime was transferred on the same day. All replicates were maintained in a climate chamber at $21125 \pm 2^{\circ} \mathrm{C}$, with a photoperiod of $16 \mathrm{~h}$ light: $8 \mathrm{~h}$ dark. During the selection experiment, all replicates 
212 in the 'Local Budding' regime were lost after generation 14, and 1 replicate in the 'Global

213 Budding' regime was lost at generation 22 (GB-3). These lines were lost because females on the

214 different patches did not produce any offspring, or only male offspring, leading to extinction of

215 lines. In total, 33 generations of selection were performed.

216

217

218

219

220

221

222

223

224

225

226

227

228

229

230

231

232

\section{Responses to selection}

\section{Sex allocation during experimental evolution}

The sex allocation of females was measured directly in the replicate populations of each selection regime at generations $12,17,20$ and 31 . This was done by counting the number of males and females per patch within each experimental replicate prior to the following transfer. Thus, sex ratio comprised the combined output of the two females per patch.

\section{Sex allocation in a common environment}

In this assay, all regimes were each exposed to a common environment for 1 generation to equilibrate maternal effects before measuring the offspring sex-ratios of females that mated randomly with males from their selection regime (Figure S1). For this, at generation 31, 96 mated daughters were haphazardly chosen from the 48 patches within each selection regime and placed on a large leaf patch $\left(\sim 200 \mathrm{~cm}^{2}\right)$ where they laid eggs together. Fourteen days later, the offspring on these patches emerged as adults and mated amongst themselves (Generation $31+1$ ). Ninetysix mated female offspring from each mixing patch were then haphazardly chosen to measure their offspring sex-ratio; 48 were placed individually on $2 \mathrm{~cm}^{2}$ patches, and another 48 placed in pairs

on $4 \mathrm{~cm}^{2}$ patches. Females were allowed to lay eggs for 7 days on these new patches, before being killed. After 2 weeks, once offspring had emerged as adults, the number of daughters and sons on 
each patch was counted. This experiment was set up over three days, with one replicate per regime

234 being treated each day.

\section{Sex allocation in response to patch fecundity}

236

237

Measures of offspring sex ratio on patches concern the sex allocation of two females on that patch.

While this is informative, it may obscure responses to selection, especially if offspring sex ratio differs between females, for instance, if a focal female's sex allocation changes in response to her own fecundity only, or also to that of her patch mate (Stubblefield \& Seger, 1990). To test this hypothesis, we measured the fecundity and sex allocation of single females from our selection regimes in response to the presence of eggs laid by sterile females on the same patch (Osouli et al., 2014). Because the eggs of the sterilised females do not hatch, we can distinguish the offspring of the focal female (adult individuals) from that of the sterilised one (unhatched eggs) within a single patch.

This experiment was implemented after 33 generations of selection. As for the preceding experiment, individuals within each replicate population were subject to a common environment. However, in this experiment it was over two generations (generation $33+2$; Figure S1). At the same time, females from the ancestral population were placed in a common environment for 2 generations, as done with females from the selection regimes (Figure S1) to generate sterile females. To sterilise these females, they were exposed to $100 \mathrm{~Gy}$, at a dose of $2.7 \mathrm{~Gy}$ minute $^{-1}$, using a Xstrahl XenX pre-clinical irradiator at the Institute of Cancer Research, Montpellier (IRCM). Preliminary studies revealed that this dose of X-ray irradiation is sufficient to sterilise females, that lay eggs that do not hatch (see Table S2). 
255

patches with one sterile female and allowed to lay eggs for 5 days. Both females were then killed and patch fecundity, the total number of eggs per patch (laid by the sterile and the fertile female, coming from the ancestral population and from one of the selection regimes, respectively) was measured. Nine days later the adult offspring of fertile females were counted, and the offspring sex ratio measured. A total of 48 leaf patches $\left(4 \mathrm{~cm}^{2}\right)$ were set up per replicate population.

\section{Sexual conflict}

The impact of mating with males evolved under the 'Global Budding' and 'Global Random' selection regimes on the fecundity of females from the ancestral population was compared in a separate assay. Females were collected from the different selection regimes at generation 33 and spent two further generations in a common environment before the experiment $(\mathrm{G} 33+2$, as above; Figure S1). The females from the ancestral population experienced one generation in a common environment, being placed in 2 boxes, each containing 100 females on a large 'mixing patch'. Thirteen days later, 240 immature, virgin females (i.e., daughters) were isolated on $4 \mathrm{~cm}^{2}$ individual leaf patches later used to measure the degree of sexual conflict.

To obtain males from each selection regime, on days 10 and 11 of the second generation in the common environment $(\mathrm{G} 33+2), 30$ immature, juvenile females were isolated from each replicate population and each placed on a $4 \mathrm{~cm}^{2}$ leaf patch. These virgin females emerged as adults and laid eggs for six days. Because spider mites are haplodiploid, only male progeny emerged from these eggs. Due to female mortality or failure to lay eggs, the total number of patches containing virgin males from each line varied from 17 to $28(\mathrm{~GB}-1=28$, GB-2 $=17$, GR-1 $=21$, GR-2 $=21$ and GR-3 = 21). On day 1 of the experiment, males from the different patches within each replicate population were mixed on a large leaf patch so they could be haphazardly distributed 
277

278

279

280

281

282

283

284

285

286

287

288

289

290

291

292

293

294

295

296

297

298

across treatments (see below).

The 240 immature, virgin females (i.e. daughters) were taken from the ancestral population and kept isolated for 2 days on their individual patches. Subsequently, the eggs laid by these females were removed and patches were assigned to males from either the 'Global Random' or 'Global Budding' selection regime, and to a 'single' or 'double' mate treatment ( $N=30$ per treatment). In all treatments, males from the selection regimes were placed with the virgin females for 5 hours on day 1 of the experiment. Twenty-four hours later (day 2), in patches assigned to the double mating treatment, a second male was placed on the patch and left for 5 hours. This mating period was chosen to take into account the time females were together with their mates each generation prior to their transfer to new patches. In both treatments, females were left to lay eggs and on day 6 of the experiment, female mortality was checked and alive females were removed from the patches. The total number of eggs per patch was counted and, 8 days later, offspring sexratio was measured.

\section{Statistical analysis}

All analyses were carried out using the R statistical package (v. 3.0.3) and JMP13. We used Generalised Linear Mixed Models (GLMMs, package glmmTMB; Brooks et al., 2017) with a betabinomial error structure and logit link function, and quasi-poisson or negative binomial error structures and log link function, to analyse the effect of selection regime on sex ratio and mean offspring production, respectively. Maximal models were simplified by sequentially eliminating non-significant terms $(\mathrm{p}<0.05)$ from the highest- to the simplest-order interaction, with the highest p-value to establish a minimal model (Crawley, 2007). The significance of the explanatory variables in the minimal models was established using chi-squared tests (Bolker et al., 2009). $A$ 
posteriori contrasts with Bonferroni corrections were done to interpret the effect of selection regime when significant (glht, multcomp package; Hothorn et al., 2008). Details of all models are given in Table S3.

\section{Sex allocation during experimental evolution}

To analyse the impact of the selection regime on offspring sex ratio, generation $(12,17,20$ and 31) and selection regime (GB, GR and LR) were included in the model as fixed factors, as well as their interaction. Generation was analysed as a covariate and was log transformed to improve the fit of the model. Experimental replicate (GB-1, GB-2, GR-1, GR-2, GR-3, LR-2 and LR-3,) was included as a random factor nested within selection regime, and the day measurements were taken as a random factor nested within generation.

\section{Sex allocation in a common environment}

To investigate the effect of selection regime on offspring sex ratio in a common environment, we used a model with selection regime (GB, GR and LR) and the number of females per patch ( 1 or 2) as fixed factors, as well as their interaction, and replicate population (GR-3, GB-1, GB-2, GR1,GR-2, LR-2 and LR-3), nested within selection regime as a random factor. This analysis excluded replicate LR-1 due to fewer than 8 patches with more than 3 offspring. For this variable, the best fit model included a parameter to account for zero inflation (ziformula 1; package glmmTMB; Brooks et al., 2017).

\section{Sex allocation in response to patch fecundity}

In a second analysis, using data from the 'Sex allocation in response to patch fecundity' experiment, we investigated whether the sex allocation of the focal female depended on her relative 
320 fecundity ('relative patch fecundity': the number of eggs laid by the focal female divided by the

321 total number of eggs laid on the patch) and on the total number of eggs present in the patch ('total

322 patch fecundity'). In this analysis, the selection regime of the focal female (GB, GR and LR),

323 relative (or total) patch fecundity were included in models as fixed factors, as well as their

324 interaction, and experimental replicate (GB-1, GB-2, GR-2, GR-3, LR-2 and LR-3) nested within

325 selection regime was added as a random factor. These analyses only included females alive on day

3264 of the experiment and excluded replicates GR-1 and LR-1, due to fewer than 10 patches with

327 more than 3 offspring.

328 We used data from this experiment to compare observed offspring sex ratios with predicted

329 values from theoretical models (see Supplementary Materials Table S1 for details) using two tailed

330 t-tests in JMP13. Observed offspring sex ratios were mean values for fertile females from each

331 selection regime.

\section{Sexual conflict}

333 To test whether selection regime affected the intensity of sexual conflict and male-male 334 competition, female fecundity and offspring sex-ratio were analysed including the number of 335 mates (one or two mates) and the selection regime of the male ('Global Budding' versus 'Global

336 Random') as discrete, fixed variables in the model, as well as their interaction. Replicate 337 population (GB-1, GB-2, GR-1, GR-2 and GR-3) and box (the container in which several 338 individual replicates were maintained) were included nested within dispersal type as random 339 factors. In the analysis of female fecundity, all individual replicates in which females died before 340 day six were excluded. 


\section{Results}

Sex allocation during experimental evolution and in a common environment

344

345

346

347

348

349

350

351

352

353

354

355

356

357

358

359

360

361

There was a consistent significant effect of selection regime on sex allocation during the selection experiment and after a generation in a common environment (during selection experiment: $\mathrm{X}^{2}{ }_{2}=$ 14.046, $\mathrm{p}<0.001$; common environment: $\mathrm{X}^{2}{ }_{2}=11.845, \mathrm{p}=0.002$; Figures $2 \mathrm{a}$ and $2 \mathrm{~b}$, Table S4). Females from the 'Global Budding' regime produced less female-biased offspring sex ratios than females from the 'Global Random' regime (during selection experiment: $Z=-3.741, \mathrm{p}<0.001$; the 'Global Budding' regime to produce a less female-biased offspring sex ratio than females from the 'Local Random' regime during the selection experiment $(Z=-2.289, \mathrm{p}=0.066)$, but not after a generation in a common environment $(Z=-1.53, \mathrm{p}=0.331$ Figures $2 \mathrm{a}$ and $2 \mathrm{~b}$; Table $\mathrm{S} 5)$. There was no difference in sex allocation between females from the 'Global Random' and 'Local Random' regimes (during selection experiment: $Z=1.554, p=0.361$; common environment: $Z=-1.597, p=$ 0.3776; Figures 2a and 2b; Table S5). The number of females on a patch did not affect offspring sex ratio (selection regime $\mathrm{x}$ number of females per patch: $\mathrm{X}^{2}{ }_{2}=4.114, \mathrm{p}=0.128$; number of females per patch: $\mathrm{X}^{2}{ }_{1}=0.94, \mathrm{p}=0.331$; Table $\mathrm{S} 4$ ).

\section{Comparing observed offspring sex-ratios with theoretical predictions}

In table S1, we present the theoretical predictions corresponding to the selection regimes in which spider mite populations evolved (Herre, 1985, Taylor \& Bulmer, 1980, Gardner et al., 2009). Females from the 'Global Random' selection regime produced an offspring sex ratio of $0.19( \pm$ $0.19 \mathrm{SE}$ ), that does not differ from the predictions of Taylor and Bulmer (1980) and of Gardner 

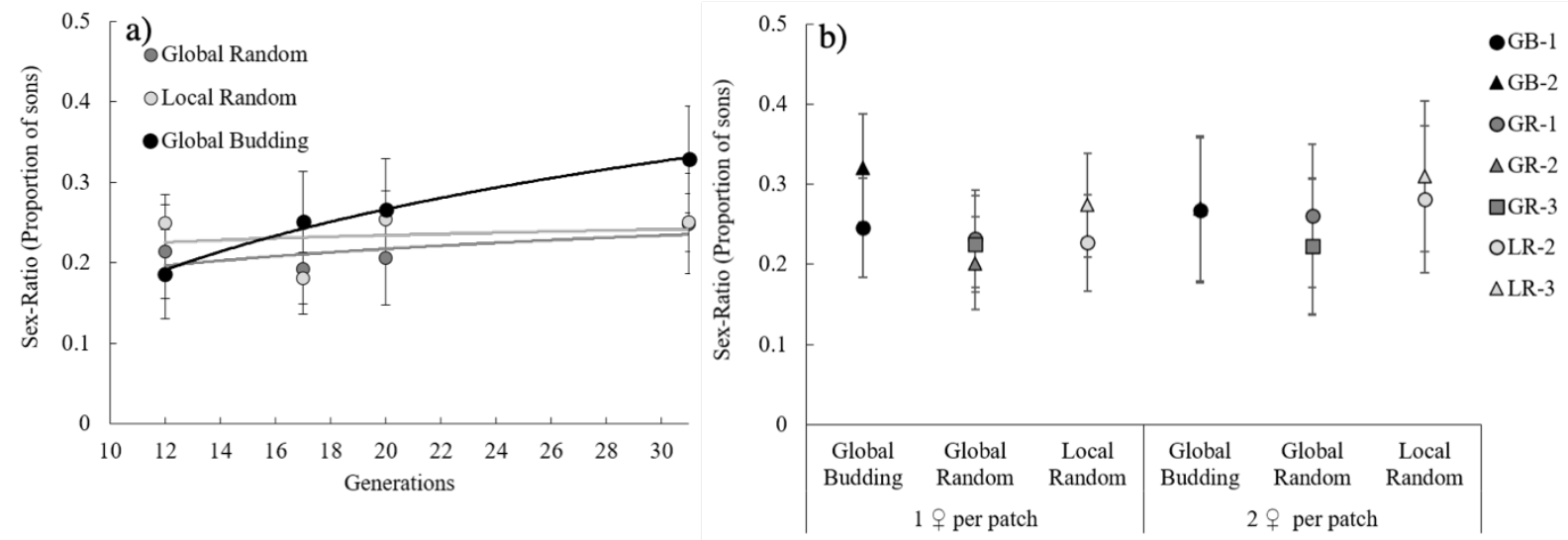

Figure 2. Mean offspring sex ratio ( \pm standard error) of females from the 'Global Random' (GR, dark grey), 'Global Budding' (GB, black) and 'Local Random' (LR, light grey) selection regimes across generations. The proportion of male offspring was measured a) during experimental evolution at generations 12,17, 20 and 31 (experimental replicate means shown for each selection regime) and b) at generation $31+1$ after one generation in a common environment, in patches with one or two females (means shown for each experimental replicate (different symbols) in each selection regime). et al (2009) $(\mathrm{t}=0.932, \mathrm{df}=69, \mathrm{p}=0.3544)$. In contrast, the evolved offspring sex ratios in the 'Global Budding' and 'Local Random' selection regimes differed from theoretical predictions. Specifically, females from the 'Global Budding' selection regime produced a less female-biased offspring sex ratio (mean $0.30 \pm 0.03 \mathrm{SE} ; \mathrm{t}=9.54$, $\mathrm{df}=55, \mathrm{p}<0.0001$ ), and females from the 'Local Random' regime a more female-biased offspring sex ratio (mean $0.24 \pm 0.02 \mathrm{SE} ; \mathrm{t}=7.99$, $\mathrm{df}=74, \mathrm{p}<0.0001)$, than predicted by theory. patch fecundity (selection regime: $\mathrm{X}_{2}{ }_{2}=10.90, \mathrm{p}=0.004$; relative patch fecundity: $\mathrm{X}^{2}{ }_{1}=6.87, \mathrm{p}=$ 

female-biased offspring sex ratio than females from the 'Global Random' regime $(Z=-3.298, p=$

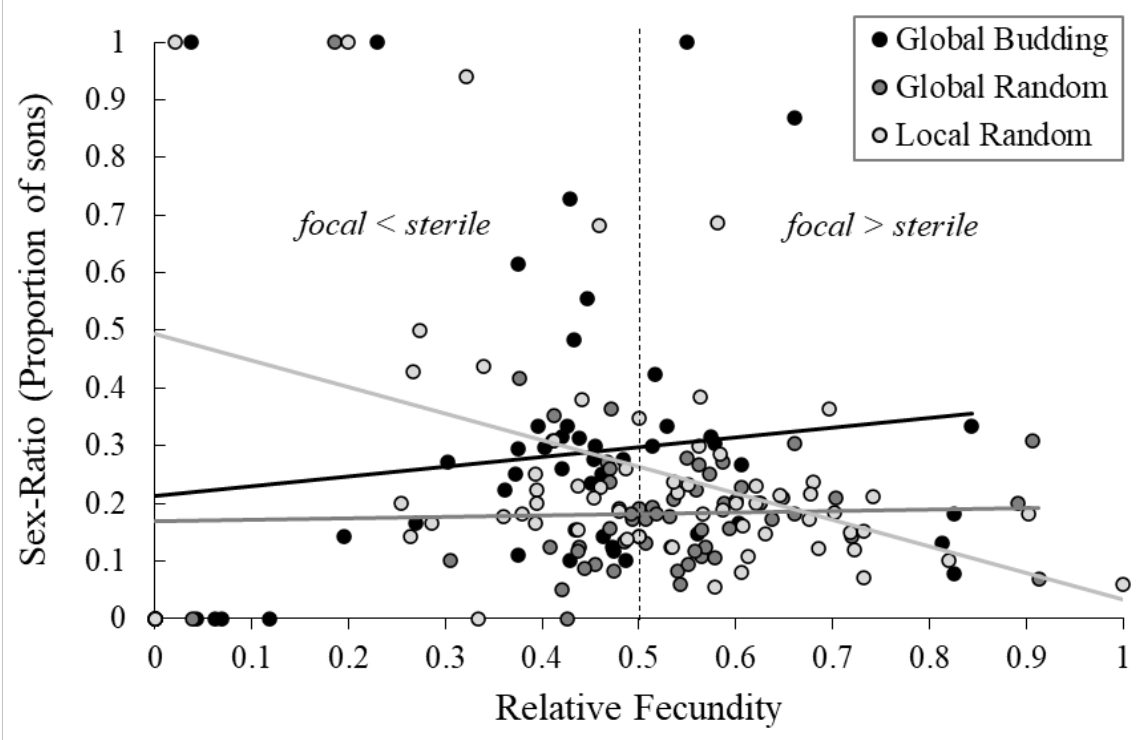

383

Figure 3. Offspring sex ratio as a function of relative patch fecundity per patch in the 'Global Budding' (GB, black), 'Global Random' (GR, dark grey) and 'Local Random' (LR, light grey) selection regimes. Females from the different selection regimes were placed on individual patches (one per patch) with a sterile female from the base population. For each patch, the proportion of offspring produced by the focal female (i.e. from the selection regime) was calculated as the proportion of eggs that hatched and became adult (relative patch fecundity), and her offspring sex-ratio was measured. Each dot represents an individual replicate (i.e., patch from which measurements were taken).

did not differ from that of the other two selection regimes (Table S5). Across all treatments, 

on each patch, Figure S2, Tables S4 and S5).

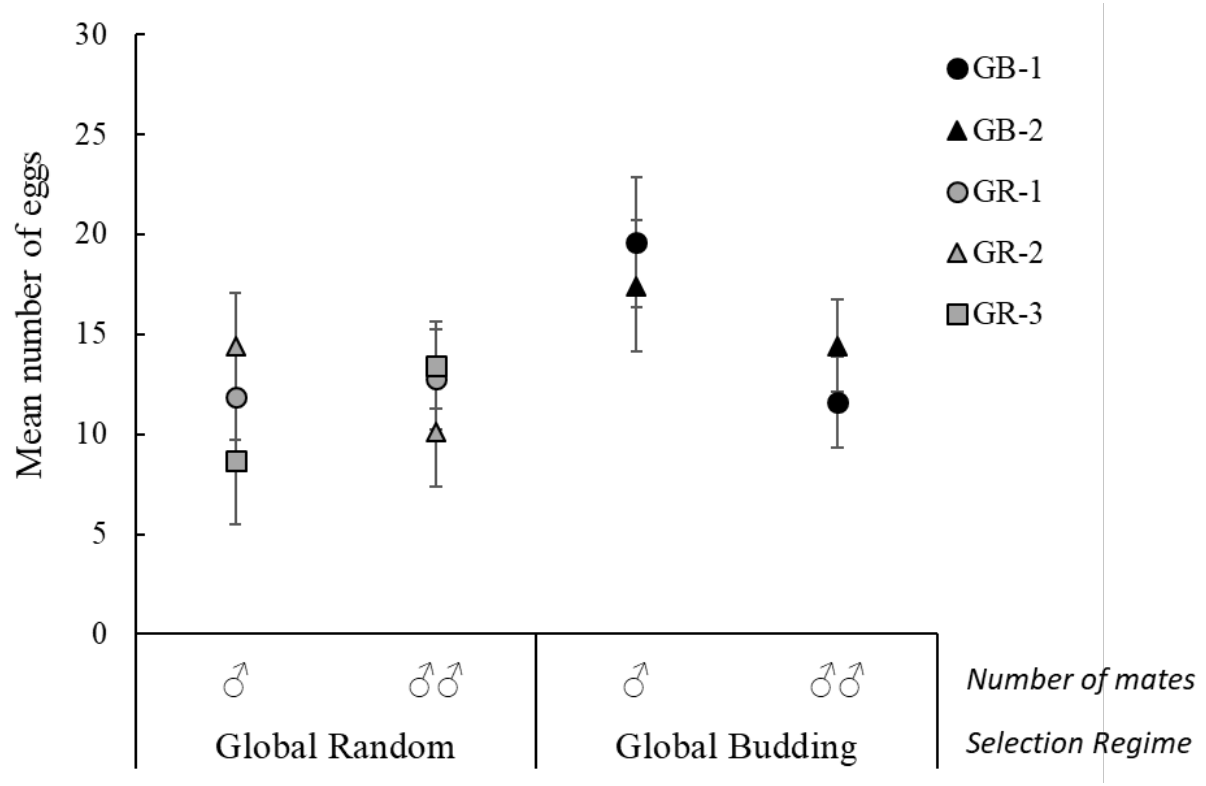

Figure 4. Mean fecundity ( \pm standard error) of females from the ancestral population presented with either one or two males from the 'Global Budding' (GB, black) or 'Global (different symbols) in each selection regime at generation 33, after two generations in a common environment.

404

Overall, there was no significant effect of mate number $\left(\mathrm{X}^{2}{ }_{1}=0.024, \mathrm{p}=0.876\right)$, male selection regime $\left(\mathrm{X}^{2}{ }_{1}=0.028, \mathrm{p}=0.867\right)$, or their interaction $\left(\mathrm{X}^{2}{ }_{1}=0.073, \mathrm{p}=0.788\right)$ on the offspring sexratio of females from the ancestral population (Figure S3, Table S4). However, the total number of offspring produced was higher when females mated with a male from the 'Global Budding', as opposed to the 'Global Random', selection regime $\left(\mathrm{X}^{2}{ }_{1}=4.336, \mathrm{p}=0.036\right.$; Figure 4, Table S4). 


\section{Discussion}

413 Both sex allocation and sexual conflict responded to selection under different population

414 structures. Sex allocation responses were mainly driven by dispersal type (budding vs random),

415 which influences whether interactions occur among kin or unrelated individuals, and not by the 416 scale of competition. Females from the 'Global Budding' regime consistently produced more 417 male-biased offspring sex ratios than those from the 'Global Random' selection regime. We also 418 found that higher relative patch fecundity was associated with more female-biased offspring sex419 ratios across all selection regimes. Finally, when comparing the intensity of sexual conflict, 420 females from the ancestral population mated to males from the 'Global Budding' regime had 421 higher fecundity than those mated to males from the 'Global Random' regime.

\section{Sex Allocation}

423 Females from the 'Global Random' selection regime produced offspring sex ratios consistent with

424 theory (Taylor \& Bulmer, 1980). This corroborates previous findings in mites (Macke et al., 2011) 425 and is consistent with sex ratio observations in other haplodiploid and diploid systems (e.g. Reece 426 et al., 2004, Reece et al., 2008, Herre, 1985). However, the 'Local Random' and the 'Global 427 Budding' regimes present offspring sex ratios that differ from theoretical predictions, being more 428 and less female-biased than predicted, respectively (see Table S1). The fact that offspring sex 429 ratios in the 'Global Budding' treatment were not as predicted, and that sex ratios in the 'Local 430 Random' and 'Global Random' regimes were equivalent, suggest that other factors, besides the 431 type of dispersal and the scale of competition, may be at play. Below, we highlight potential factors 432 that may account for the observed patterns. 
First, inbreeding is affected by dispersal type (with high inbreeding expected for the

434

435

436

437

438

439

440

441

442

443

444

445

446

447

448

449

450

451

452

453

454

455

budding dispersal regime). Inbreeding can select for more female-biased offspring sex ratios

(Herre, 1985, Chung et al., 2019, Frank, 1985). If coupled with high levels of juvenile mortality this could, in some cases, result in no males in a patch (Chung et al., 2019, West et al., 2002b), potentially explaining why all 3 replicates of the 'Local Budding' and 1 replicate of the 'Global Budding' regimes were lost. Moreover, different inbreeding levels may lead to different levels of inbreeding depression among selection regimes, being highest under budding dispersal. Thus, in our surviving selection regimes, the consequences of inbreeding depression may be more pronounced in the 'Global Budding' regime. In haplodiploids like spider mites, inbreeding depression is expressed mainly in female traits (Tien et al., 2015). Therefore, the accrued costs of inbreeding may negate any benefit of female-biased sex ratios in the 'Global Budding' regime in the replicates that survived (Greeff, 1996). As such, there might be selection to augment the production of sons in patches with low fecundity and/or high mortality due to inbreeding depression to ensure female fertilisation (Chung et al., 2019, West et al., 2002b). In line with this, females in the 'Global Budding' regime produced fewer offspring that became adult, which might be due to higher offspring mortality or lower fecundity (Figure S4, Table S5). These females also produced slightly more male offspring than those from the 'Global Random' regime (Figure S5a, Table S5).

Another factor that may affect sex allocation is variable clutch size. Indeed, when females with asymmetric fecundities oviposit simultaneously in the same patch, the sons of a more fecund female are subject to more intense competition for mates, as they mostly compete among brothers to mate with sisters, whereas the sons of a less fecund female mostly compete with unrelated males to access unrelated females (Stubblefield \& Seger, 1990). More fecund females are thus expected 
456 to produce more female-biased sex ratios, while less fecund females should produce less female-

457 biased sex ratios. As a result, the patch sex ratio becomes skewed towards that produced by the

458 more fecund females, i.e. a more female-biased sex ratio (Stubblefield \& Seger, 1990, West, 2009).

459 In addition, theory predicts that this adjustment by females should emerge from a differential

460 investment in daughters, while maintaining a constant production of sons, known as the "constant

461 male hypothesis' (Yamaguchi, 1985, Frank, 1987). Here, we found that, for all selection regimes,

462 the sex ratio declined as the relative fertility of the focal female increased (the same was observed

463 for total patch fecundity, Figure S2), showing that female fecundity and sex-ratio are not

464 independent traits. Furthermore, although not significantly different from the other regimes, 'Local

465 Random' females generated the steepest slope. Coupled with higher overall fecundity observed in

466 this selection regime (Figure S4, Table S5), this may explain why offspring sex ratios are more

467 female-biased than expected. Finally, although son production is not constant across selection

468 regimes (Figure S5a, Table S4), its variation is much lower than that for the number of daughters

469 produced (Figure S5b). Again, this seems to be in line with an effect of clutch size on sex

470 allocation. Note however that the constant male hypothesis is expected to break down under local

471 competition (Rodrigues \& Gardner, 2015), a result we do not recapitulate here.

472 A final possibility for why populations in the 'Local Random' and 'Global Budding' 473 selection regimes do not produce the predicted offspring sex ratios is that they have not reached

474 their evolutionary equilibrium. Another selection experiment found an initial reduction in

475 cooperation under budding dispersal followed by a subsequent increase in this trait value

476 (Kummerli et al., 2009). Although our experimental evolution was done over 33 generations,

477 which is a large number for non-microbial organisms, populations may still have been in a transient 478 stage when traits were measured. 


\section{Sexual Conflict}

480 Theory predicts that multiple mating with unrelated males causes a greater reduction in female 481 reproductive success than multiple mating with related males (Pizzari et al., 2015). More precisely, 482 Faria et al (2020) predict that budding dispersal leads to higher relatedness between males, thus 483 reducing levels of sexual conflict and increasing levels of female fecundity. In accordance with 484 this we found that females mated to males from the 'Global Budding' regime had higher fecundity than those mated to males from the 'Global Random' regime, suggesting that 'Global Budding' 486 males inflicted less harm. This replicates previous findings showing that evolving with kin reduced 487 the level of male harm inflicted to females in bulb mites (Lukasiewicz et al., 2017). Other studies 488 have shown that a reduction in male harm may be a plastic response to the presence of kin (Carazo 489 et al., 2014, Lymbery \& Simmons, 2017). However, in our experiment, since mating was with unrelated females from the ancestral population, there were no direct cues indicating the presence of kin. In addition, competitor males coming from the same selection regime experienced 2 generations of common garden prior to the experiment which probably reduced relatedness among them. This means that if the response were plastic, then there should be no difference between selection regimes. Thus, reduced harm was most likely an evolved response in our study. reduced fecundity in either selection regime. Possibly, the differences in harm inflicted by one or two mates over a single, or two successive five-hour periods respectively, might have been 498 insufficient to detect differences in fecundity between the two treatments. Previous studies with spider mites found fecundity costs when females were simultaneously exposed to multiple mates

500 for two consecutive periods of 24 hours with an interval of $24 \mathrm{~h}$ hours between them (Rodrigues et 501 al., 2020), or exposed to two mates on multiple occasions during their lifetime (Macke et al., 2012). 
Here we only tested the effect of the type of dispersal on sexual conflict. However, the

503

504

505

506

507

508

509

510

511

512

513

514

515

516

517

518

519

520

521

522

523

outcome of sexual conflict may also change according to the type of population regulation. Indeed, under local competition, increased competition among relatives is predicted to cancel out the benefits of cooperation (Queller, 1992, Taylor, 1992, Wilson et al., 1992). This means that sexual conflict might be maintained among related individuals when competition is local (Pizzari et al., 2015, Wild et al., 2011). Yet, despite its general interest, we are not aware of any studies that explicitly test this.

\section{The interplay between sex allocation and sexual conflict}

Evolution under different population structures may simultaneously impact sex allocation and sexual conflict in a non-independent manner (Chapman, 2009, Schärer \& Janicke, 2009). One possibility is that sexual conflict might impact sex allocation if a reduction in female fecundity prevents the production of optimal offspring sex ratios. Our sexual conflict experiment showed that females from the ancestral population mated to males from the 'Global Random' regime had the lowest fecundity, suggesting that these males inflict more harm. Yet females from the 'Global Random' regime produced offspring sex ratios closest to those predicted by theory (Table S1). In addition, 'Global Random' females, when mated to 'Global Random' males in the sex allocation experiment had higher fecundity (Figure S4, Table S4). This suggests that 'Global Random' females may have evolved mechanisms to overcome male harassment or induced harm, as shown in this (Macke et al., 2014) and other (Wigby \& Chapman, 2004, Michalczyk et al., 2011) systems. Female resistance to harassment may thus be one trait involved simultaneously in the outcome of sexual conflict and sex allocation.

Conversely, sex allocation may also impact sexual conflict through changes in levels of 
524 male-male competition; as the sex ratio becomes more male biased so will the intensity of

525 competition. Indeed, evolving with kin may reduce male harm and be associated with more female-

526 biased offspring sex ratios (Lukasiewicz et al., 2017); although the latter was not significantly

527 different from the non-kin evolution treatment). In our sex allocation experiment, sex ratio was the

528 least female-biased in the 'Global Budding' selection regime. However, males from this regime

529 inflicted the least harm to females from the ancestral population (sexual conflict experiment), 530 suggesting sex allocation evolution did not result in stronger sexual conflict.

\section{Conclusions}

532 To date, we are only aware of one study that considers the evolution of both sex allocation and 533 sexual conflict under different population structures (Lukasiewicz et al., 2017). Although the latter 534 study is a very important step toward integrating responses of both traits, relatedness was the only 535 variable that differed between treatments. Therefore, much remains to be investigated concerning 536 the consequences of populations structure for these traits, which have been found to be non537 independent in a number of systems (Macke et al., 2014, Boulton et al., 2019, Boulton \& Shuker, 538 2015). Here, we show that responses to selection on these traits under different population 539 structures mostly depended on the dispersal regime. Clearly, more studies simultaneously 540 investigating the evolution of sex allocation and sexual conflict are needed, as interactions among 541 these traits may account for data not matching theoretical predictions.

542

\section{Acknowledgements}

544 We would like to thank François Rousset and Elsa Noël for helpful discussion about this 545 manuscript. This work was funded by an ERC consolidating grant (COMPCON GA 725419) to 
546 SM, a joint grant from the Agence Nationale de la Recherche and the Fundação para a Ciência e a

547 Tecnologia to Isabelle Olivieri and SM (FCT-ANR/BIA- EVF/0013/2012), a PHC-PESSOA grant 548 (38014YC) to ABD and SM, and a SIRIC Montpellier Cancer Grant 549 (INCa_Inserm_DGOS_12553) funded the irradiator. This is ISEM contribution number 2020-310.

550

551 Data is archived at Dryad: https://doi.org/10.5061/dryad.w3r2280ph

552

553 
555 Arnqvist, G. \& Rowe, L. 2005. Sexual Conflict. Princeton University Press, Princeton.

556 Aviles, L. 1993. Interdemic selection and the sex ratio: A social spider perspective. Am. Nat. 142: 320$557 \quad 345$.

558 Bolker, B. M., Brooks, M. E., Clark, C. J., Geange, S. W., Poulsen, J. R., Stevens, M. H. \& White, J. S. 559 2009. Generalized linear mixed models: a practical guide for ecology and evolution. Trends Ecol 560 Evol 24: 127-35.

561

562

563

564

565

566

567

568

569

570

571

572

573

574

575

576

577

578

Boulton, R. A., Cook, N., Greenway, E. V., Glaser, G. L., Green, J. \& Shuker, D. M. 2019. Local mate competition modifies the costs of mating in a mostly monandrous parasitoid wasp. Behav Ecol 30: 417-425.

Boulton, R. A. \& Shuker, D. M. 2015. A sex allocation cost to polyandry in a parasitoid wasp. Biol Lett 11: 20150205.

Bourke, A. F. 2009. The kin structure of sexual interactions. Biol Lett 5: 689-92.

Breeuwer, J. A. 1997. Wolbachia and cytoplasmic incompatibility in the spider mites Tetranychus urticae and T. turkestani. Heredity 79: 41-47.

Brooks, M. E., Kristensen, K., van Benthem, K. J., Magnusson, A., Nielson, A., Skuag, H. J., Maechler, M. \& Bolker, B. M. 2017. Balances Speed and Flexibility among Packages for Zero-Inflated Generalized Linear Mixed Modeling. The R Journal 9: 41-47.

Bulmer, M. G. 1986. Sex ratio theory in geographically structured populations. Heredity 56: 69-73.

Carazo, P., Tan, C. K., Allen, F., Wigby, S. \& Pizzari, T. 2014. Within-group male relatedness reduces harm to females in Drosophila. Nature 505: 672-5.

Chapman, T. 2009. Sexual conflict and sex allocation. Biol Lett 5: 660-2.

Charnov, E. L. 1982. The Theory of Sex Allocation. Princeton University Press, Princeton.

Chippindale, A. K., Berggren, M., Alpern, J. H. \& Montgomerie, R. 2015. Does kin selection moderate sexual conflict in Drosophila? Proc R Soc Lond B 282: 20151417. 
579

580

581

582

583

584

585

586

587

588

589

590

591

592

593

594

595

596

597

598

599

600

601

602

603

604

Chung, N., Pienaar, J. \& Greeff, J. M. 2019. Evolutionary stable sex ratios with non-facultative male-eggs first sex allocation in fig wasps. Oikos 128: 859-868.

Courteau, J. \& Lessard, S. 2000. Optimal sex ratios in structured populations. J Theor Biol 207: 159-75.

Crawley, M. J. 2007. The R Book. Wiley.

Crudgington, H. S., Beckerman, A. P., Brustle, L., Green, K. \& Snook, R. R. 2005. Experimental removal and elevation of sexual selection: does sexual selection generate manipulative males and resistant females? Am Nat 165: S72-S87.

Eldakar, O. T. \& Wilson, D. S. 2011. Eight criticisms not to make about group selection. Evolution 65: 1523-6.

Faria, G. S., Gardner, A. \& Carazo, P. 2020. Kin discrimination and demography modulate patterns of sexual conflict. Nat Ecol Evol 4: 1141-1148.

Faria, G. S., Varela, S. A. \& Gardner, A. 2015. Sex-biased dispersal, kin selection and the evolution of sexual conflict. J Evol Biol 28: 1901-10.

Frank, S. 1985. Hierarchical selection theory and sex ratios. II. On applying the theory, and a test with fig wasps. Evolution 39: 949-964.

Frank, S. 1986. The genetic value of sons and daughters. Heredity 56: 351-354.

Frank, S. 1987. Variable sex ratio among colonies of ants. Behav Ecol Sociobiol 20: 195-201.

Frank, S. 1998. Foundations of social evolution. Princeton University Press, Princeton.

Gardner, A., Arce, A. \& Alpedrinha, J. 2009. Budding dispersal and the sex ratio. J Evol Biol 22: 1036-45.

Gardner, A. \& West, S. A. 2006. Demography, altruism, and the benefits of budding. J Evol Biol 19: 170716.

Greeff, J. M. 1996. Alternative mating strategies, partial sibmating and split sex ratios in haplodiploid species. J Evol Biol9: 855-869.

Griffin, A. S., West, S. A. \& Buckling, A. 2004. Cooperation and competition in pathogenic bacteria. Nature 430: 1024-1027.

Hamilton, W. D. 1964. The genetical evolution of social behaviour. I. J Theor Biol 7: 1-16. 
605

606

607

608

609

610

611

612

613

614

615

616

617

618

619

620

621

622

623

624

625

626

627

628

629

Hamilton, W. D. 1967. Extraordinary sex ratios. Science 156: 477-488.

Helle, W. \& Sabelis, M. W. 1985. Spider Mites: Their Biology, Natural Enemies and Control. Elsevier, Amsterdam.

Herre, E. A. 1985. Sex ratio adjustment in fig wasps. Science 228: 896-898.

Hollis, B., Kawecki, T. J. \& Keller, L. 2015. No evidence that within-group male relatedness reduces harm to females in Drosophila. Ecol Evol 5: 979-83.

Hothorn, T., Bretz, F. \& Westfall, P. 2008. Simultaneous inference in general parametric models. Biomet $J$ 50: $346-363$.

Kennedy, G. G. \& Smitley, D. R. (1985) Dispersal. In: Spider mites. Their biology, natural enemies and control. Vol. 1. pp. Elsevier, Amsterdam.

Krainacker, D. A. \& Carey, J. R. 1989. Reproductive limits and heterogeneity of male two-spotted spider mites. Entom Exp et Appl 50: 209-214.

Kummerli, R., Gardner, A., West, S. A. \& Griffin, A. S. 2009. Limited dispersal, budding dispersal, and cooperation: an experimental study. Evolution 63: 939-49.

Le Page, S., Sepil, I., Flintham, E., Pizzari, T., Carazo, P. \& Wigby, S. 2017. Male relatedness and familiarity are required to modulate male-induced harm to females in Drosophila. Proc Roy Soc Lond B 201: 20170441.

Lehmann, L., Perrin, N. \& Rousset, F. 2006. Population demography and the evolution of helping behaviors. Evolution 60: 1137-1151.

Lehmann, L. \& Rousset, F. 2010. How life history and demography promote or inhibit the evolution of helping behaviours. Philos Trans R Soc Lond B 365: 2599-2617.

Lukasiewicz, A., Szubert-Kruszynska, A. \& Radwan, J. W. 2017. Kin selection promotes female productivity and cooperation between the sexes. Sci Adv3: e1602262.

Lymbery, S. J. \& Simmons, L. W. 2017. Males harm females less when competing with familiar relatives. Proc Roy Soc Lond B 284: e20171984. 
Macke, E., Magalhães, S., Bach, F. \& Olivieri, I. 2011. Experimental evolution of reduced sex ratio adjustment under local mate competition. Science 334: 1127-1129.

Macke, E., Magalhães, S., Do-Thi Khanh, H., Frantz, A., Facon, B. \& Olivieri, I. 2012. Mating modifies female life history in a haplodiploid spider mite. Am Nat 179: E147-162.

Macke, E., Olivieri, I. \& Magalhães, S. 2014. Local mate competition mediates sexual conflict over sex ratio in a haplodiploid spider mite. Curr Biol 24: 2850-4.

Michalczyk, L., Millard, A. L., Martin, O. Y., Lumley, A. J., Emerson, B. C. \& Gage, M. J. 2011. Experimental evolution exposes female and male responses to sexual selection and conflict in Tribolium castaneum. Evolution 65: 713-724.

Migeon, A. \& Dorkeld, F. (2019) Spider Mites Web: A Comprehensive Database for the Tetranychidae. https://www1.montpellier.inra.fr/CBGP/spmweb/.

Mitchell, R. 1973. Growth and population dynamics of a spider mite (Tetranychus urticae K., Acarina: Tetranychidae). Ecology 54: 1349-1355.

Osouli, S., Nejad, K. H. I., Ziaie, F. \& Moghaddam, M. 2014. Gamma irradiation used on adult Tetranychus urticae Koch as a quarantine treatment. J Plant Prot Res 54: 150-155.

Parker, G. A. 2006. Sexual conflict over mating and fertilization: an overview. Philos Trans R Soc Lond B 361: 235-59.

Pizzari, T., Biernaskie, J. M. \& Carazo, P. 2015. Inclusive fitness and sexual conflict: How population structure can modulate the battle of the sexes. BioEssays 37: 155-166.

Pizzari, T. \& Gardner, A. 2012. The sociobiology of sex: inclusive fitness consequences of inter-sexual interactions. Philos Trans R Soc Lond B 367: 2314-23.

Queller, D. C. 1992. Does population viscosity promote kin selection? Trends Ecol Evol 7: 322-324.

Rankin, D. J. 2011. Kin selection and the evolution of sexual conflict. J Evol Biol 24: 71-81.

Reece, S. E., Drew, D. R. \& Gardner, A. 2008. Sex ratio adjustment and kin discrimination in malaria parasites. Nature 453: 609-14. 
Reece, S. E., Shuker, D. M., Pen, I., Duncan, A. B., Choudhary, A., Batchelor, C. M. \& West, S. A. 2004. Kin discrimination and sex ratios in a parasitoid wasp. J Evol Biol 17: 208-216.

Rodrigues, A. M. M. \& Gardner, A. 2015. Simultaneous failure of two sex-allocation invariants: implications for sex-ratio variation within and between populations. Proc Roy Soc Lond B 282.

Rodrigues, L. R., Figueiredo, A. R. T., Van Leeuwen, T., Olivieri, I. \& Magalhães, S. 2020. Costs and benefits of multiple mating in a species with first-male sperm precedence. J Anim Ecol 89: 10451054.

Rousset, F. 2004. Genetic structure and selection in subdivided populations. Princeton University Press, Princeton.

Schärer, L. \& Janicke, T. 2009. Sex allocation and sexual conflict in simultaneously hermaphroditic animals. Biol Lett 5: 705-708.

Stubblefield, J. W. \& Seger, J. 1990. Local mate competition with variable fecundity: dependence of offspring sex ratios on information utilization and mode of male production. Behav Ecol 1: 68-80.

Taylor, P. D. 1981. Intra-sex and intersex sibling interactions as sex ratio determinants. Nature 291: 64-66.

Taylor, P. D. 1992. Altruism in viscous populations - an inclusive fitness model. Evol Ecol 6: 352-356.

Taylor, P. D. \& Bulmer, M. G. 1980. Local mate competition and the sex ratio. J Theor Biol 86: 409-419.

Taylor, P. D. \& Crespi, B. J. 1994. Evolutionary stable strategy sex ratios when correlates of relatedness can be assessed. Am Nat 143: 297-316.

Tien, N. S., Sabelis, M. W. \& Egas, M. 2015. Inbreeding depression and purging in a haplodiploid: genderrelated effects. Heredity 114: 327-32.

West, S. A. 2009. Sex Allocation. Princeton University Press, Princeton.

West, S. A., Pen, I. \& Griffin, A. S. 2002a. Cooperation and competition between relatives. Science 296: $72-5$.

West, S. A., Smith, T. G. \& Read, A. F. 2002b. Fertility insurance and the sex ratios of malaria and related hemosporin blood parasites. J Parasit 88: 258-263. 
680 Wigby, S. \& Chapman, T. 2004. Female resistance to male harm evolves in response to manipulation of $681 \quad$ sexual conflict. Evolution 58: 1028-1037.

682 Wild, G., Pizzari, T. \& West, S. A. 2011. Sexual conflict in viscous populations: the effect of the timing of 683 dispersal. Theor Popul Biol 80: 298-316.

684 Wilson, D. S., Pollock, G. B. \& Dugatkin, L. A. 1992. Can altruism evolve in purely viscous populations? 685 . Evolutionary Ecology 6: 331-341.

686 Yamaguchi, Y. 1985. Sex ratios of an aphid subject to local mate competition with variable maternal 687 condition. Nature 318: 460-462.

688 Zélé, F., Santos, I., Matos, M., Weill, M., Vavre, F. \& Magalhães, S. 2020. Endosymbiont diversity in 689 natural populations of Tetranychus mites is rapidly lost under laboratory conditions. Heredity 124: $690 \quad 603-617$.

691 Zélé F., Santos, I., Olivieri, I., Weill, M., Duron, O. \& Magãlhaes, S. 2018. Endosymbiont diversity and 692 prevalence in herbivorous spider mite populations in South-Western Europe. FEMS Microbiol Ecol 693 94.

694 